\title{
Estructura de la comunidad de esponjas (Porifera) en tres arrecifes del Parque Nacional Morrocoy, Venezuela y su relación con algunas variables ambientales
}

\author{
Marco A. Romero ${ }^{1}$, Estrella Villamizar ${ }^{2}$ \& Nora Malaver ${ }^{2}$ \\ 1. Escuela de Biología, Facultad de Ciencias, Universidad Central de Venezuela, Caracas, Venezuela; \\ elmarcuspe@hotmail.com \\ 2. Instituto de Zoología y Ecología Tropical, Facultad de Ciencias, Universidad Central de Venezuela, Caracas, \\ Venezuela; estrella.villamizar@ciens.ucv.ve,nora.malaver@ciens.ucv.ve
}

Recibido 06-VIII-2012. Corregido 10-I-2013. Aceptado 24-I-2013.

\begin{abstract}
Community structure of sponges (Porifera) in three reefs at Morrocoy National Park, Venezuela and its correspondence with some environmental variables. Sponges have an important ecological role in coral reef ecosystems. However, when compared to other benthic Phyla, it has been little researched. This research was focused in the variability of the community structure of sponges in three locations at Morrocoy National Park (Cayo Sombrero, Playa Mero and Punta Brava) exposed to different environmental conditions (transparency and currents intensity) and affected in different degree of severity by a mass mortality event in 1996. A total of 15 transects ( $10 \mathrm{~m}$ long and $1 \mathrm{~m}$ wide) were evaluated in three strata (between 3 and $15 \mathrm{~m}$ depth) in each site, where all the individuals were counted by species. Relative abundance by species, diversity and evenness were calculated. Locations showed differences respect turbidity, wave and current intensity. 27 species were found in Morrocoy; Cayo Sombrero (23), Playa Mero (18) and Punta Brava (15). Agelas sceptrum, Amphimedon erina and Niphates erecta were the most common in first location; Niphates erecta and Dysidea etheria in Playa Mero and Dysidea etheria, Niphates erecta and Amphimedon erina in Punta Brava. The species composition showed statistical differences between all three locations; Cayo Sombrero resulted the most diverse and even, followed by Playa Mero and Punta Brava. According to Sorensen Similarity Index results, Cayo Sombrero and Playa Mero were more similar, while Punta Brava resulted the most different. The variability in environmental conditions and the differential mass mortality effects of 1996 in all three reefs, were probably the main causes of the differences between their sponge communities. Nevertheless, we cannot conclude about the weight of these factors. Rev. Biol. Trop. 61 (3): 1229-1241. Epub 2013 September 01.
\end{abstract}

Key words: sponges communities, Morrocoy National Park, species composition, environmental variables, mass mortality event.

Las esponjas juegan una importante función ecológica en los arrecifes coralinos. Además de competir por el espacio con los demás organismos sésiles en este ecosistema, son fuente de refugio y alimento para numerosos vertebrados e invertebrados marinos, como juveniles de langosta, peces, poliquetos, moluscos, ofiuroideos, entre otros (Rützler 1978, Villamizar 1985, Butler et al. 1995). De igual forma, algunas especies pueden contribuir a la consolidación de los arrecifes coralinos creciendo entre las colonias de coral y “cementándolas" (Díaz \& Rützler 2001), mientras que otras contribuyen a su erosión aportando sedimentos finos de origen coralino (Rützler 1978, McKenna 1997, Díaz \& Rützler 2001, Chaves-Fonnegra et al. 2005). No obstante, el esfuerzo de investigación dedicado a las esponjas a nivel mundial es mucho menor en referencia a otros grupos de organismos marinos, por lo que muchos temas de estudio relacionados con este Phyllum quedan aún por desarrollar.

En Venezuela se han realizado algunos trabajos a lo largo de la costa continental y en las 
áreas insulares. Olivares (1976) realizó un estudio en la bahía de Mochima en el cual reportó 12 especies de esponjas. Para el Parque Nacional Morrocoy, Díaz et al. (1985) señalaron 23 especies en las aguas internas, sobre las raíces de Rhizophora mangle. En el Parque Nacional Archipiélago Los Roques, fueron reportadas 33 especies por Álvarez \& Díaz (1985) y posteriormente, tres especies nuevas de la familia Demospongiae por Díaz et al. (1987). Pauls (1998) reportó 40 especies para la Ciénaga de Ocumare de la Costa; Amaro \& Liñero (2002) reportaron 11 especies para una localidad dentro del Parque Nacional Mochima, señalando posteriormente 22 especies en el mencionado Parque (Amaro \& Liñero 2006). Alvizu (2006) reportó para la Isla de Cubagua, Estado Nueva Esparta 31 especies, y finalmente en Isla Larga, Parque Nacional San Esteban fueron reportadas 17 especies por Núñez et al. (2010).

Este trabajo se desarrolló durante el año 2002, en el Parque Nacional Morrocoy, situado en Golfo Triste, costa del Estado Falcón. En este parque existen dos zonas marinas: la zona externa a los cayos, de franca influencia oceánica, caracterizada por mostrar un desarrollo importante de comunidades coralinas, oleaje moderado, turbidez baja y profundidades de hasta 20 metros, y la zona interna, más cercana a la costa y protegida por los cayos, que a su vez se caracteriza por tener un oleaje menor, mayor turbidez y aguas someras con desarrollo de manglares, principalmente $R$. mangle y lechos de fanerógamas marinas (Losada \& La Schiazza 1989).

En Morrocoy, si bien se tiene conocimiento de las comunidades de esponjas para las aguas internas, se desconocen las comunidades de poríferos que ocupan los hábitats de arrecifes coralinos de las aguas externas, donde existen distintas condiciones ambientales a lo largo de los arrecifes franjeantes y cayos (Solana et al. 2004). Unido a esto, la zona externa del parque fue objeto de un evento de mortalidad masiva en 1996, que afectó de forma diferencial los distintos arrecifes, y que ocasionó una notable disminución de la cobertura coralina viva, lo que afectó otro grupos tales como a esponjas, poliquetos, gasterópodos, equinodermos, peces entre otros (Villamizar 2000, Laboy-Nieves et al. 2001). Desde el punto de vista ecológico, esta situación reviste mucha importancia para los estudios que se llevan a cabo de forma posterior a este evento.

El objetivo de este estudio fue conocer la estructura de las comunidades de poríferos en tres sitios del Parque Nacional Morrocoy, que presentan diferentes condiciones ambientales y grados de afectación por el evento de mortalidad masiva. Se espera que la estructura de las comunidades de esponjas de las tres localidades de estudio esté relacionada con tales condiciones.

\section{MATERIALES Y MÉTODOS}

Área de estudio: Los muestreos se desarrollaron entre mayo y noviembre de 2002 en tres sitios arrecifales, en las localidades de Cayo Sombrero, Playa Mero y Punta Brava (Fig. 1), que se describen a continuación. El sitio de Cayo Sombrero está ubicado en el canal situado entre el mencionado cayo y tierra firme, con profundidades entre los tres y $15 \mathrm{~m}$, una corriente con dirección predominante hacia el norte-noroeste paralela a la costa continental y a la del cayo, con valores que llegan a alcanzar los $40 \mathrm{~cm} / \mathrm{s}$ (Fig. 1), siendo los máximos registrados para Morrocoy (Solana et al. 2004). La influencia del oleaje en el sitio no se considera importante por estar situado el mismo a sotavento del cayo (Solana et al. 2004).

Playa Mero, localizada en Cayo de Ánimas, constituye un arrecife franjeante protegido por una pequeña ensenada con una profundidad máxima de $15 \mathrm{~m}$. Los datos de corrientes para esta estación provienen de mediciones realizadas fuera de la ensenada, pues en su interior el efecto de la corriente es mucho menor. La dirección registrada es norte-noreste (Fig. 1), con valores entre 2 y $6 \mathrm{~cm} / \mathrm{s}$ (Solana et al. 2004). Respecto al oleaje, el sitio de muestreo se encuentra protegido por la ensenada y su impacto no es relevante, sin embargo para las aguas externas existen datos procedentes de mediciones tomadas en Boca 


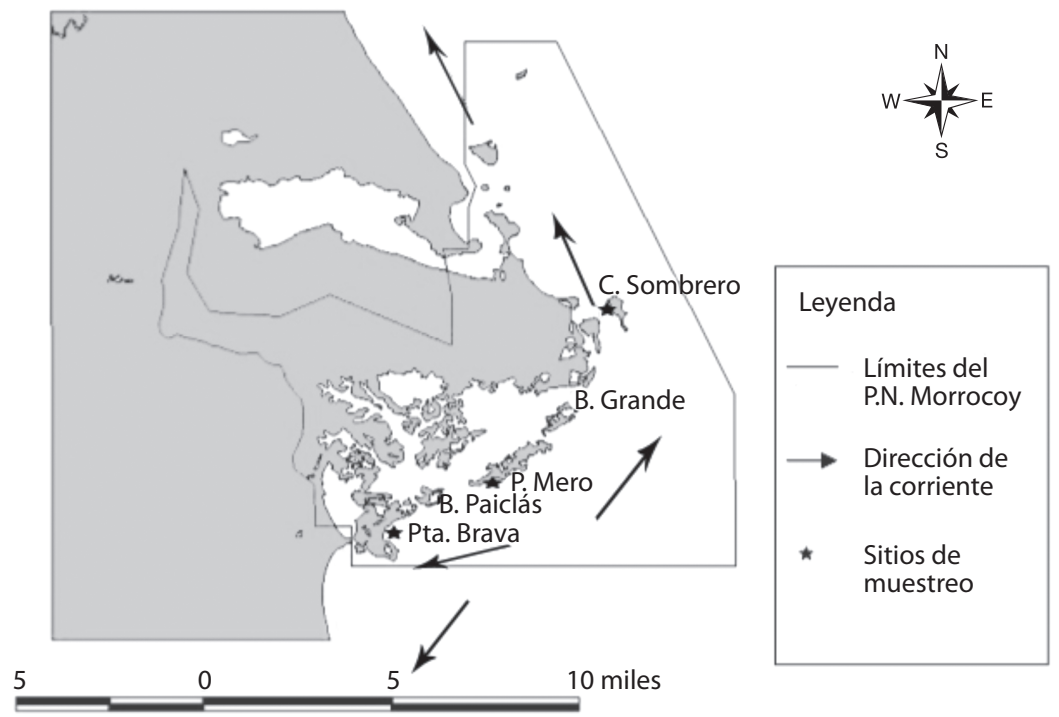

Fig. 1. Parque Nacional Morrocoy, donde se indican la dirección de las corrientes, los sitios de muestreos y los dos sitios donde se obtuvieron mediciones de oleaje (Boca Paiclás y Boca Grande).

Fig. 1. Morrocoy National Park. Arrows shows current direction, stars shows the study sites. "B. paiclás" and "B. Grande" were sites of wave measurements.

Paiclás y Boca Grande (Fig. 1), los cuales presentan comportamientos similares en cuanto a período y altura del oleaje, situación que es más o menos parecida a lo largo de la zona del parque expuesta al mar abierto. El período de la ola se caracteriza por oscilar entre cuatro y seis segundos, con una altura de ola de $1 \mathrm{~m}$, típico del oleaje originado por la acción del viento local, registrándose períodos mayores solo en algunas ocasiones, lo que indica la ocurrencia de oleajes de tormenta (Solana et al. 2004).

Por su parte, el arrecife de Punta Brava, localizado en el Cayo del mismo nombre, se caracteriza por ser un arrecife franjeante similar al de Playa Mero, en el cual el desarrollo coralino se observa hasta una profundidad máxima de $12 \mathrm{~m}$. A diferencia de los sitios anteriores, está completamente expuesto al oleaje. La corriente medida en una zona situada frente a la localidad de estudio presenta una dirección predominante oeste-suroeste (Fig. 1) con una magnitud fluctuante entre 2 y $6 \mathrm{~cm} / \mathrm{s}$ (Solana et al. 2004). Esta localidad, según Martín (2005), está influenciada por dos factores importantes, la cercanía a una playa con gran desarrollo turístico y la influencia cercana de dos ríos importantes que desembocan en Golfo Triste, el Aroa y el Yaracuy.

Muestreo de las comunidades de esponjas: La evaluación de las comunidades de poríferos se llevó a cabo por el método de transectos lineales de ancho fijo. Para ello se identificaron y contaron todas las esponjas presentes a lo largo de transectos de $10 \mathrm{~m}$ de largo y $1 \mathrm{~m}$ de ancho, dispuestos en dirección paralela a la costa y separados entre sí por una distancia de 10m (la ubicación del primer transecto se realizó en forma aleatoria). Con la única finalidad de obtener una representación del arrecife en toda su extensión, se colocaron cinco transectos en cuatro estratos de profundidad: Estrato uno: entre tres y seis metros, Estrato dos: entre seis y nueve metros, Estrato tres: entre nueve y $12 \mathrm{~m}$ y Estrato cuatro: entre 12 y $15 \mathrm{~m}$. En los arrecifes de Cayo Sombrero y Playa Mero se muestreó un área total de $200 \mathrm{~m}^{2}$ (cinco transectos por cuatro estratos de profundidad), mientras que en el arrecife de Punta Brava solo fue posible muestrear un área de $150 \mathrm{~m}^{2}$, ya que 
la máxima profundidad de este arrecife en el sitio de estudio es de $12 \mathrm{~m}$.

Para cada especie de esponja en cada localidad estudiada, se calculó la abundancia relativa, en base al número total de esponjas en cada unidad de muestreo (transecto). Solo para Cliona aprica se midió el porcentaje de cobertura lineal en lugar de la abundancia, ya que la forma incrustante de esta especie y su amplia cobertura sobre esqueletos de Acropora palmata, impidieron el conteo de los individuos. Cuando la identificación de las especies se dificultó en el campo, se tomaron pequeñas muestras de las esponjas para su posterior identificación en el laboratorio según Zea (1987) y Hooper \& van Soest (2002).

Se calculó el índice de Sorensen (1948), con el fin de determinar la similitud en la composición de especies entre las comunidades de esponjas estudiadas. De esta forma, se calculó la similitud entre las tres comunidades, de manera simultánea y entre cada par de comunidades por separado. Su expresión matemática es la siguiente: $\mathrm{C}_{\mathrm{S}}=2 \mathrm{~A} / 2 \mathrm{~A}+\mathrm{B}+\mathrm{C}+\mathrm{D}$, siendo $\mathrm{A}=$ número de especies comunes a las tres (o dos) comunidades, $\mathrm{B}=$ número de especies exclusivas de la comunidad uno, $\mathrm{C}=$ número de especies exclusivas de la comunidad dos y $\mathrm{D}=$ número de especies exclusivas de la comunidad tres. Los valores calculados para este índice oscilan entre cero y uno. Adicionalmente se calcularon los índices de Diversidad expresados en nits/individuo (Shannon-Wiener) y equidad (Pielou 1966) para cada comunidad. Se empleó la prueba estadística t para comparar los valores del índice de diversidad según plantea Magurran (1988), empleando el software estadístico Past 1.95.

Se infirieron las condiciones ambientales para las tres comunidades de poríferos estudiadas. Esto se hizo según la relación establecida por Alcolado (1992) posteriormente modificado (Alcolado 1999); entre los valores de diversidad (H') y equidad (J'), obtenidos a partir del análisis de 104 estudios sobre comunidades de esponjas en diversos biotopos y su asociación con las características ambientales de cada uno de ellos, como se aprecia en el diagrama de la figura 2. Las inferencias sobre el ambiente marino se refieren al grado de benignidad o severidad del mismo, así como su comportamiento temporal (pronosticabilidad) para la fauna sésil. Para inferir las condiciones ambientales de las comunidades de poríferos aquí estudiadas, se extrapolaron sus valores de H'y J' en el citado diagrama.

Para comparar la estructura de las tres comunidades de esponjas estudiadas, se empleó un análisis de ordenamiento por escalamiento multidimensional no paramétrico (MDS, por sus siglas en inglés), donde las muestras corresponden a los transectos realizados en cada sitio y se representan como puntos en un espacio bidimensional, correspondiéndose a las distancias relativas entre los puntos con las disimilitudes relativas (Bray-Curtis), según Clarke \& Warwick (2001). Adicionalmente a esto, se realizó un análisis de varianza multivariado en base a permutaciones (PERMANOVA) empleando 9999 permutaciones (Anderson 2001, McArdle \& Anderson 2001) para evaluar el factor sitio, dada la no normalidad en la distribución de los datos y la heterogeneidad en la varianza de los mismos. Se tomó como hipótesis nula que las comunidades de esponjas son similares en los tres sitios evaluados. Debido a que en Punta Brava no se realizó el estrato correspondiente a los $15 \mathrm{~m}$ de profundidad, este fue eliminado en las otras localidades a los efectos de hacer el muestreo balanceado, situación que se requiere para esta prueba. Finalmente, se realizó el análisis SIMPER (Porcentajes de similitud-contribución por especies), para determinar cuáles especies son las que más aportan a que las unidades de muestreo (transectos) en cada localidad sean parecidas entre sí, así como cuáles especies contribuyen a la disimilitud entre las localidades (Clarke \& Warwick 2001), seleccionando en cada caso, aquellas especies cuya contribución a la similitud o disimilitud en porcentajes, sea igual o superior a 10. Las pruebas MDS y SIMPER se realizaron empleando el software estadístico PRIMER 5.

Los datos correspondientes a las variables ambientales -temperatura, salinidad, oxígeno 


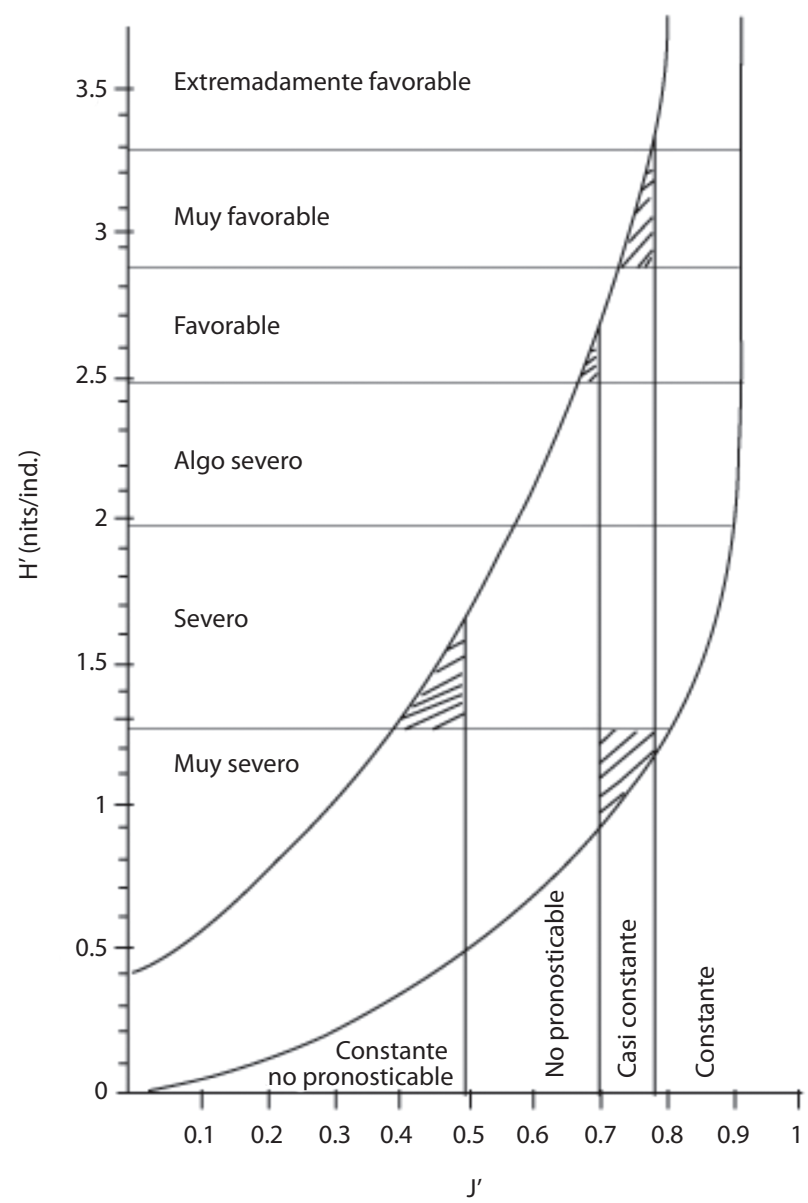

Fig. 2. Diagrama modificado de inferencias de severidad y pronosticabilidad ambiental para las comunidades de esponjas según Alcolado (1999), a partir de valores de diversidad ( $\left.\mathrm{H}^{\prime}\right)$ y valores de equidad (J').

Fig. 2. Modified diagram for severity inferences and environmental prognostic in sponge communities after Alcolado (1999), considering diversity $\left(\mathrm{H}^{\prime}\right)$ and equity values (J').

disuelto, transparencia y sólidos totales disueltos (SST) en el agua-, fueron tomadas de Martín (2005), quien realizó registros mensuales de las mismas entre los años 2000 y 2001. Dado que existe normalidad y homogeneidad de varianza, un ANOVA fue empleado para comparar las mediciones mencionadas entre las localidades estudiadas y determinar si existen diferencias significativas entre ellas.

\section{RESULTADOS}

Composición de especies: Fueron identificadas un total de 27 especies agrupadas en 12 familias, ocho órdenes y dos subclases, pertenecientes a la clase Demospongiae. En Cayo Sombrero se encontró el mayor número de especies (23), seguido por Playa Mero (18) y Punta Brava (15) (Cuadro 1). Respecto al número de individuos totales, fue en Cayo Sombrero donde se obtuvo el mayor valor con 613, seguido por Playa Mero con 497 y finalmente Punta Brava con 455. En Playa Mero, la especie Cliona aprica presentó un porcentaje de cobertura lineal promedio de $11.40 \%$ (media $=11.40, \mathrm{DE}=5.67, \mathrm{n}=5$ ) entre los tres $\mathrm{y}$ seis metros, sobre los esqueletos de Acropora palmata, mientras que en Punta Brava el valor 
CUADRO 1

Listado taxonómico de las especies encontradas en las localidades estudiadas.

Clasificación tomada de Hooper \& van Soest (2002)

TABLE 1

Taxonomic list of species found at the study sites

PHYLUM PORIFERA Grant, 1836

CLASE DEMOSPONGIAE Sollas, 1888

SUBCLASE CERACTINOMORPHA Lévi, 1953

ORDEN DICTYOCERATIDA Minchin, 1900

FAMILIA THORECTIDAE Bergquist, 1978

Ircinia felix (Duchassaing \& Michelotti, 1864)

Ircinia strobilina (Lamark, 1816)

Smenospongia aurea (Hyatt, 1875) •

FAMILIA DYSIDEIDAE Gray, 1867

Dysidea etheria de Laubenfels, $1936 \bullet \mathbf{\Delta}$

ORDEN HALISARCIDA Bergquist 1996

FAMILIA HALISARCIDAE

Halisarca $\mathrm{sp} \bullet \mathbf{\Delta} \mathbf{\square}$

ORDEN VERONGIDA Bergquist, 1978

FAMILIA APLYSINIDAE Carter, 1875

Aiolochroia crassa (Hyatt, 1875) • $\mathbf{\Delta}$.

Aplysina archeri (Higgin, 1875) $\bullet \mathbf{\Delta}$ -

Aplysina cauliformis (Carter, 1882) $\bullet \boldsymbol{\Delta} \boldsymbol{\square}$

Aplysina fistularis (Pallas, 1766)

Aplysina lacunosa (Lamark, 1814) • $\boldsymbol{\Delta}$

Verongula rigida (Esper, 1794) $\bullet \boldsymbol{\Delta}$

ORDEN HAPLOSCLERIDA Topsent, 1928

FAMILIA NIPHATIDAE van Soest, 1980

Amphimedon erina (de Laubenfels, 1936) •

Niphates erecta Duchassaing \& Michelotti, 1864

$\bullet \boldsymbol{\Delta}$ -

FAMILIA CALLYSPONGIIDAE de Laubenfels, 1936 Callyspongia vaginalis (Lamark, 1814) • $\boldsymbol{\Delta}$

FAMILIA PETROSIIDAE van Soest, 1980 Neopetrosia subtriangularis (Duchassaing, 1850) Neopetrosia proxima (Duchassaing \& Michelotti, 1864)

Xestospongia muta (Schmidt, 1870) •

ORDEN POECILOSCLERIDA Topsent, 1928

FAMILIA ESPERIOPSIDAE Hentschel, 1923

Desmapsamma anchorata (Carter, 1882) • $\boldsymbol{\Delta}$.

Iotrochota birotulata (Higgin, 1877) $\bullet \mathbf{\Delta}$ -

FAMILIA MICROCIONIDAE Carter, 1875

Clathria sp • A

ORDEN HALICHONDRIDA Gray, 1867

FAMILIA DICTYONELLIDAE van Soest, Díaz \&

Pomponi 1990

Scopalina hispida (Hechtel, 1965)

Scopalina ruetzleri (Wiedenmayer, 1977) • $\boldsymbol{\Delta}$ -

ORDEN AGELASIDA Hartman, 1980

FAMILIA AGELASIDAE Verrill, 1907

Agelas clathrodes (Schmidt, 1870) • A

Agelas dispar Duchassaing \& Michelotti, 1864 •

Agelas sceptrum (Lamark, 1815) $\bullet \boldsymbol{\Delta}$

Agelas schmidti Wilson, $1902 \bullet \boldsymbol{A}$
SUBCLASE TETRACTINOMORPHA Lévi, 1953

ORDEN HADROMERIDA Topsen, 1894

FAMILIA CLIONIDAE D'Orbigny, 1851

Cliona aprica Pang, 1973

La presencia de la especie en cada localidad se representa con el símbolo correspondiente: Cayo Sombrero $(\bullet)$, Playa Mero ( $\mathbf{\Lambda})$ y Punta Brava (๘).

fue de $21.20 \%$ (media $=21.20, \mathrm{DE}=8.48, \mathrm{n}=5$ ) en las mismas condiciones, no encontrándose esta especie en el estrato somero de Cayo Sombrero, donde no existió coral muerto (sustrato cubierto por Millepora sp.). Como puede observarse en el cuadro 1, las comunidades de esponjas de los tres arrecifes estudiados tienen en común un gran número de especies. Los resultados obtenidos mediante la aplicación del Índice de Similitud de Sorensen corroboran esta observación. Así, el índice señala un 74\% de similitud en la composición de especies entre las tres comunidades. Por su parte, al comparar la similitud entre la comunidad de esponjas de Cayo Sombrero y Playa Mero, el valor fue de $83 \%$, entre las comunidades de Cayo Sombrero y Punta Brava fue de 67\%, y entre las de Punta Brava y Playa Mero de 61\%.

Los tres sitios del Parque Nacional Morrocoy evaluados mostraron diferencias respecto a la composición y abundancia relativa de las diferentes especies. El resultado del MDS (Fig. 3) mostró que los transectos pertenecientes a las diferentes localidades se agruparon de manera diferencial. Estos resultados se corroboraron con el análisis PERMANOVA, en el que se apreciaron diferencias significativas entre las localidades $(\mathrm{p}<0.05)$. Según el análisis SIMPER, las especies Agelas sceptrum con 29.63\% de contribución, Amphimedon erina con $18.91 \%$ y Niphates erecta con $10.78 \%$ fueron las que más contribuyeron a que las muestras (transectos) de Cayo Sombrero resultaran más similares entre sí. En el caso de Playa Mero fueron $N$. erecta con $61.61 \%$ y Dysidea etheria con $19.37 \%$, mientras que para Punta Brava D. etheria presentó $35.14 \%, N$. erecta $30.65 \%$ y $A$. erina $21.43 \%$ (Cuadro 2). Estas 


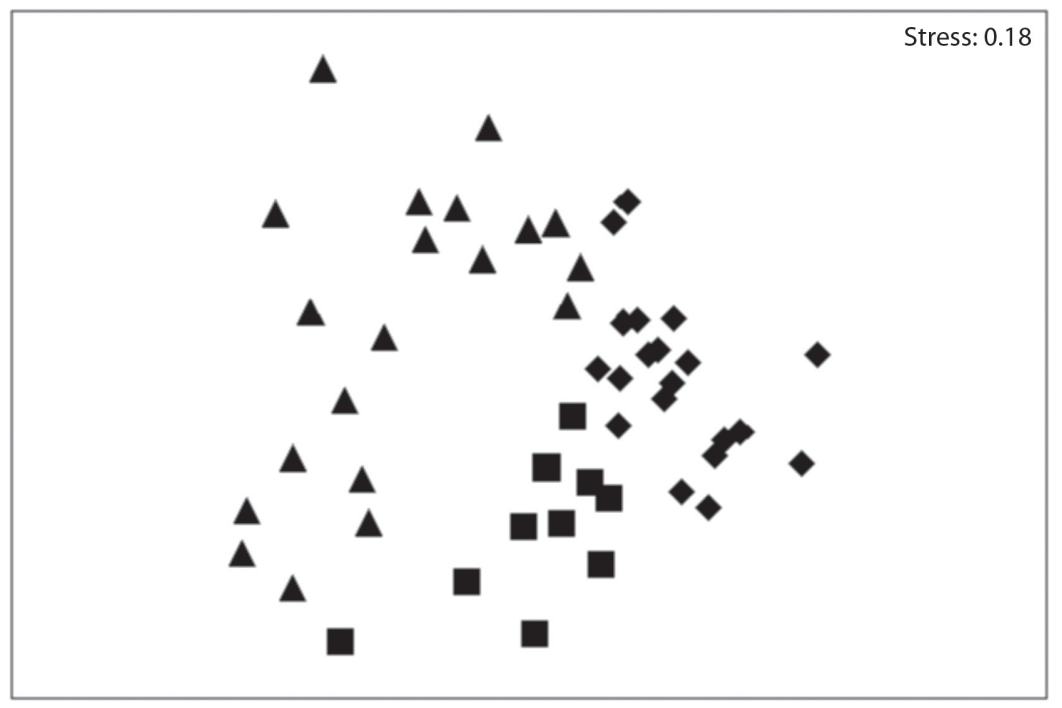

Fig. 3. Resultados del MDS (Análisis de ordenamiento por escalamiento multidimensional no paramétrico) que muestra el agrupamiento diferencial de los transectos en cada localidad en base a la abundancia relativa de las especies. $\boldsymbol{\Lambda}$ : Cayo Sombrero, : Playa Mero, a: Punta Brava.

Fig. 3. Multidimensional scaling analysis showing differential grouping of transect from each site based on relative abundance of sponge species. $\mathbf{\Delta}$ : Cayo Sombrero, $\$$ : Playa Mero, n: Punta Brava.

CUADRO 2

Análisis SIMPER (Porcentajes de similitud-contribución por especies) para detectar las especies responsables de la similitud entre las unidades de muestreo dentro de cada localidad

\section{TABLE 2}

SIMPER analysis for detection of species contribution to the similarity between transects inside each site

\begin{tabular}{lcc}
$\begin{array}{l}\text { Especies para } \\
\text { cada localidad }\end{array}$ & $\begin{array}{c}\text { Porcentaje de } \\
\text { contribución a la } \\
\text { similitud (\%) } \\
\text { Cayo Sombrero }\end{array}$ & $\begin{array}{c}\text { Porcentaje } \\
\text { acumulado (\%) }\end{array}$ \\
A. sceptrum & 29.63 & 29.63 \\
A. erina & 18.91 & 48.55 \\
N. erecta & 10.78 & 59.33 \\
& Playa Mero & \\
N. erecta & 61.61 & 61.61 \\
D. etheria & 19.37 & 80.98 \\
& Punta Brava & 35.14 \\
D. etheria & 35.14 & 65.79 \\
N. erecta & 30.65 & 87.21 \\
A. erina & 21.43 & \\
\hline
\end{tabular}

Solo se tomaron las especies que contribuyeron en más de un $10 \%$ al porcentaje de similitud. especies a su vez coincidieron en cada localidad con las que presentaron mayores porcentajes de abundancia relativa (Cuadro 3).

Este mismo análisis, pero al evaluar el aporte de las distintas especies a la disimilitud entre localidades (Cuadro 4), mostró para el par Cayo Sombrero-Playa Mero, que las especies que más contribuyeron a las diferencias observadas fueron: $N$. erecta $(20.67 \%)$, A. erina $(12.17 \%)$, D. etheria $(11.67 \%)$ y $A$. sceptrum (11.13\%). Entre Cayo Sombrero y Punta Brava, las especies que más aportaron a las diferencias fueron: $D$. etheria $(16.93 \%), A$. erina $(16.80 \%), N$. erecta $(14.85 \%)$ y $A$. sceptrum $(12.45 \%)$, mientras que entre Playa Mero y Punta Brava, las que más influyeron en la disimilitud fueron: $N$. erecta $(20.74 \%)$, A. erina $(18.99 \%)$ y $D$. etheria $(18.33 \%)$.

Diversidad, equidad e inferencias acerca de las condiciones ambientales: Cayo Sombrero fue la que presentó mayores valores de diversidad y equidad (2.64nits/ind. y 0.88 , respectivamente), seguido por Playa Mero 
CUADRO 3

Abundancia relativa y desviación estándar de las distintas especies en cada localidad

TABLE 3

Relative abundance and standard deviation of the species at each site

\begin{tabular}{|c|c|c|c|c|c|}
\hline Especies & $\begin{array}{c}\text { C. Sombrero } \\
\text { (\% A.R.) }\end{array}$ & Especies & $\begin{array}{l}\text { P. Mero } \\
\text { (\% A.R.) }\end{array}$ & Especies & $\begin{array}{l}\text { P. Brava } \\
\text { (\% A.R.) }\end{array}$ \\
\hline A. sceptrum & $19.42 \pm 10.38$ & N. erecta & $36.36 \pm 11.62$ & D. etheria & $18.84 \pm 18.32$ \\
\hline A. erina & $18.91 \pm 26.06$ & D. etheria & $19.47 \pm 4.58$ & N. erecta & $17.93 \pm 15.53$ \\
\hline$N$. erecta & $9.13 \pm 6.78$ & S. ruetzleri & $8.54 \pm 4.07$ & A. erina & $15.02 \pm 20.14$ \\
\hline A. crassa & $8.00 \pm 7.40$ & Halisarca sp. & $6.95 \pm 4.65$ & I. birotulata & $3.55 \pm 6.14$ \\
\hline I. felix & $6.45 \pm 10.91$ & A. sceptrum & $5.50 \pm 3.88$ & I. strobilina & $2.53 \pm 2.64$ \\
\hline A. archeri & $6.24 \pm 2.69$ & I. strobilina & $4.08 \pm 1.68$ & A. cauliformis & $1.94 \pm 3.36$ \\
\hline A. clathrodes & $5.80 \pm 5.98$ & A. archeri & $3.54 \pm 3.83$ & I. felix & $1.88 \pm 2.90$ \\
\hline I. birotulata & $3.80 \pm 1.49$ & A. clathrodes & $3.54 \pm 3.79$ & S. hispida & $1.14 \pm 1.97$ \\
\hline A. cauliformis & $3.09 \pm 3.89$ & A.cauliformis & $1.66 \pm 1.38$ & Halisarca $\mathrm{sp}$. & $1.05 \pm 1.82$ \\
\hline D. anchorata & $2.95 \pm 2.98$ & C. vaginalis & $1.11 \pm 2.22$ & D. anchorata & $0.73 \pm 1.26$ \\
\hline I. strobilina & $2.84 \pm 1.42$ & A. crassa & $1.04 \pm 1.16$ & A. fistularis & $0.61 \pm 0.53$ \\
\hline C. vaginalis & $2.69 \pm 2.42$ & I. birotulata & $0.91 \pm 1.06$ & A. archeri & $0.47 \pm 0.43$ \\
\hline D. etheria & $2.52 \pm 0.83$ & A. lacunosa & $0.90 \pm 1.31$ & A. crassa & $0.38 \pm 0.65$ \\
\hline Halisarca sp. & $1.75 \pm 3.11$ & Clathria sp & $0.54 \pm 0.65$ & S. ruetzleri & $0.37 \pm 0.44$ \\
\hline A. dispar & $1.73 \pm 3.45$ & D. anchorata & $0.51 \pm 0.56$ & N. proxima & $0.11 \pm 0.19$ \\
\hline S. aurea & $1.27 \pm 2.54$ & A. schmidti & $0.38 \pm 0.76$ & N. subtriangularis & $0.11 \pm 0.19$ \\
\hline A. lacunosa & $1.12 \pm 1.18$ & $V$. rigida & $0.24 \pm 0.47$ & & \\
\hline
\end{tabular}

En Cayo Sombrero solo se representan las especies con porcentaje de abundancia relativa superior a 1.

\section{CUADRO 4}

Análisis SIMPER para detectar las especies responsables de la disimilitud entre las localidades estudiadas

TABLE 4

SIMPER analysis for detection of species contribution to the dissimilarity between study sites

\begin{tabular}{lcc}
$\quad \begin{array}{c}\text { Comparación } \\
\text { entre localidades }\end{array}$ & $\begin{array}{c}\text { Porcentaje de contribución } \\
\text { a la disimilitud (\%) } \\
\text { Cayo Sombrero - Playa Mero }\end{array}$ & $\begin{array}{c}\text { Porcentaje } \\
\text { acumulado (\%) }\end{array}$ \\
N. erecta & 20.67 & 20.67 \\
A. erina & 12.17 & 32.85 \\
D. etheria & 11.67 & 44.52 \\
A. sceptrum & 11.13 & 55.65 \\
& Cayo Sombrero - Punta Brava & 16.93 \\
D. etheria & 16.93 & 33.73 \\
A. erina & 16.80 & 48.58 \\
N. erecta & 14.85 & 61.02 \\
A. sceptrum & 12.45 & \\
& Playa Mero - Punta Brava & 20.74 \\
N. erecta & 20.74 & 39.72 \\
A. erina & 18.99 & 58.06 \\
D. etheria & 18.33 & \\
\hline
\end{tabular}

Solo se tomaron las especies que contribuyeron en más de un $10 \%$ a la disimilitud entre pares de comunidades. 
CUADRO 5

Valor promedio y desviación estándar de las variables ambientales (Tomadas de Martín 2005)

TABLA 5

Mean and standard deviation of environmental variables

\begin{tabular}{lccccc}
\multicolumn{1}{c}{ Localidad } & Temperatura $\left({ }^{\circ} \mathrm{C}\right)$ & Salinidad $(\%)$ & Turbidez $(\mathrm{m})$ & SST $(\mathrm{mg} / \mathrm{L})$ & Oxígeno disuelto $(\mathrm{mg} / \mathrm{L})$ \\
Cayo Sombrero & $27.91 \pm 2.41$ & $38.22 \pm 2.94$ & $8.04 \pm 2.25$ & $103.38 \pm 144.73$ & $5.50 \pm 0.91$ \\
Playa Mero & $28.28 \pm 1.23$ & $38.14 \pm 2.95$ & $6.22 \pm 3.14$ & $87.00 \pm 67.24$ & $5.74 \pm 0.95$ \\
Punta Brava & $28.99 \pm 1.25$ & $36.74 \pm 2.83$ & $2.32 \pm 0.77$ & $108.45 \pm 155.29$ & $5.59 \pm 0.88$ \\
\hline
\end{tabular}

En itálicas aparece la variable que presentó diferencias significativas $(\mathrm{p}<0.05)$ entre localidades.

(diversidad: 2.07nits/ind. y equidad: 0.75) y Punta Brava (diversidad: 1.85nits/ind. y equidad: 0.71) en ese orden. La prueba t realizada para comparar los valores de diversidad demostró que las diferencias observadas son significativas $(\mathrm{p}<0.05)$.

Al emplear el método descrito por Alcolado (1992) y modificado por el propio autor (Alcolado 1999), se pudo inferir las condiciones ambientales: en el caso de Cayo Sombrero como "favorables y constantes", en Playa Mero resultaron "algo severas y casi constantes" y en Punta Brava "severas y casi constantes".

Variables ambientales: Solo la transparencia presentó diferencias significativas entre las tres localidades (ANOVA $\mathrm{p}<0.05$; Cuadro 5), la cual junto a la corriente y a la acción del oleaje fueron las que marcaron las diferencias en lo que a las condiciones ambientales se refiere.

\section{DISCUSIÓN}

La comunidad de esponjas asociada a los tres sitios estudiados en el Parque Nacional Morrocoy presentó cierta similitud entre sí (Índice de Sorensen), respecto a la composición de especies. Sin embargo, diferencias notables fueron encontradas respecto a la abundancia relativa y la representación de dichas especies dentro de cada localidad. De esta forma, entre Cayo Sombrero y Playa Mero las especies que más contribuyeron a las diferencias fueron $N$. erecta, A. erina, D. etheria y A. sceptrum. La segunda especie no se observó en Playa Mero, aunque si fue muy común en Cayo Sombrero, mientras que $N$. erecta y $D$. etheria fueron dominantes en Playa Mero y más escasas en Cayo Sombrero, ocurriendo a la inversa con $A$. sceptrum. Entre Cayo Sombrero y Punta Brava, las más contribuyentes a la disimilitud fueron D. etheria, A. erina, $N$. erecta, y A. sceptrum. Punta Brava está dominada por las especies $D$. etheria y $N$. erecta, especies que son menos abundantes en Cayo Sombrero, mientras que $A$. sceptrum es la especie dominante en esta localidad, estando ausente en Punta Brava. Pese a estar bien representada en las dos localidades, A. erina es más abundante en Cayo Sombrero, aunque su distribución es más homogénea en Punta Brava, contribuyendo más que en Cayo Sombrero a la similitud entre transectos. Respecto a Playa Mero y Punta Brava, $N$. erecta, $A$. erina y $D$. etheria son las que marcan la diferencia. En Playa Mero N. erecta es la especie más abundante y mejor distribuida en todas las unidades de muestreo, contribuyendo en un $61.61 \%$ a la similitud entre las mismas, mientras que en Punta Brava, esta especie es menos abundante y de distribución más heterogénea en el arrecife. A la inversa ocurre con $D$. etheria, mientras que $A$. erina está ausente en Playa Mero y resultó abundante en Punta Brava.

Comparando las especies presentes en el Parque Nacional Morrocoy con las registradas en el Parque Nacional Archipiélago Los Roques por Díaz et al. (1985), encontramos que la mayoría de las especies reportadas en este trabajo están presentes también en Los Roques, a 
diferencia de Smenospongia aurea (extremadamente escasa en Morrocoy y solo registrada en Cayo Sombrero), D. etheria, Halisarca sp., A. erina, Desmapsamma anchorata, Clathria sp. y Scopalina híspida (solo registrada en Punta Brava sobrecreciendo bivalvos). De estas especies $D$. etheria fue reportada por Aerts (1998) como muy abundante en arrecifes de la costa colombiana en comparación con los arrecifes de Curazao, lo que pudiera significar que esta especie es típica de arrecifes continentales y no de los arrecifes insulares alejados de la influencia de tierra firme (variaciones de salinidad, turbidez, aporte de nutrientes, sedimentación, entre otros).

De las tres comunidades estudiadas en este trabajo, Playa Mero y Punta Brava estuvieron dominadas mayormente por tres especies: $N$. erecta, $D$. etheria y $A$. erina, presentándose esta última especie solo en la segunda localidad mencionada. La dominancia de estas tres especies podría indicar la mejor adaptación de las mismas a un factor estresante. Sará (1970) y Alcolado (1999) manifestaron que las esponjas tienden más a cooperar entre sí que a competir, sin embargo, ciertos factores ambientales condicionan la proliferación de algunas especies sobre otras. Tal es el caso de las que no presentan endosimbiontes fotosintéticos que le aportan nutrientes y dependen totalmente de la filtración para obtener alimento (totalmente heterótrofas), las cuales son más favorecidas en ambientes de aguas turbias que aquellas que dependen más de sus simbiontes (Valderrama \& Zea 2003). Según Erwin \& Thacker (2007), estas especies presentan muy bajos contenidos de Clorofila $a$ en sus tejidos, lo cual puede ser indicativo de ausencia o muy poca presencia de simbiontes fotosintetizadores, resultados confirmados para $D$. etheria, en la cual estos autores no reportaron presencia de cianobacterias por medio de análisis de reacción en cadena de la polimerasa (PCR, en inglés) específicos para ellas.

De estas tres especies, $N$. erecta domina -al igual que $D$. etheria- en ambientes caracterizados por salinidad fluctuante y elevada turbidez. Es muy abundante en el Golfo de Urabá,
Colombia (Valderrama y Zea 2003) y la Isla Nelson en Trinidad y Tobago (Hubbard 1990). En Playa Mero ambas presentan una abundancia relativa total de $55.83 \%$, mientras que en Punta Brava, añadiendo a $A$. erina totalizan un $51.79 \%$. Esto significa que ellas representan aproximadamente la mitad del total de individuos de las distintas especies contabilizadas en los mencionados sitios. El mismo patrón fue observado en el Golfo de Urabá, donde dominaron las especies $N$. erecta, Iotrochota birotulata y Mycale laevis -todas heterótrofas- en un $45 \%$ sobre el resto (Valderrama \& Zea 2003) y en Los Roques, donde las especies dominantes representaron el $60 \%$ del total de la comunidad en aguas sujetas a descargas de sedimentos provenientes de la laguna interna del archipiélago, que generan condiciones de elevada turbidez (Álvarez et al. 1990).

Una situación diferente fue observada en Cayo Sombrero, donde las especies con mayor abundancia relativa fueron $A$. sceptrum y $A$. erina, con menor presencia de $N$. erecta. Las dos primeras especies totalizan un 38\% del total de individuos de la comunidad. Esta tendencia se observa en arrecifes que presentan condiciones óptimas para el desarrollo de las esponjas, como el Archipiélago de San Andrés, donde las especies dominantes representaron el $25 \%$ de la comunidad (Valderrama \& Zea 2003). Cayo Sombrero además es la localidad que presenta un mayor número de especies y los valores más altos de diversidad y equidad. Esta localidad presenta condiciones de baja turbidez comparado con Playa Mero y Punta Brava, además de presentar fuertes corrientes que favorecen el crecimiento de las esponjas al incrementar el flujo de agua que se establece a través del organismo (Bell \& Barnes 2000), sin que los valores lleguen a ser extremos, lo que constituiría un factor de estrés mecánico sobre los organismos.

Las condiciones ambientales inferidas a partir de los valores de diversidad y equidad, siguiendo el método descrito por Alcolado (1992) muestran que la localidad donde las condiciones resultaron más idóneas fue Cayo Sombrero (condiciones "favorables y 
constantes"). La presencia de fuertes corrientes, relativamente baja turbidez, influencia nula del oleaje (el arrecife esta a sotavento del cayo) y salinidad óptima, sin muchas variaciones (al menos durante el período muestreado) permitió el desarrollo de una comunidad de esponjas diversa y más equitativa que en las otras dos localidades.

Por su parte Playa Mero se catalogó como un ambiente "algo severo y casi constante". Este arrecife presentó valores de turbidez más altos que Cayo Sombrero y a su vez las corrientes fueron considerablemente inferiores. La escasa corriente y la poca influencia del oleaje probablemente dificultaron la remoción de los sedimentos que usualmente se depositan sobre esponjas y corales, observándose en el momento del muestreo, muchas esponjas cubiertas totalmente de sedimento. Está situación reduce la capacidad de filtración en estos organismos, retardando su crecimiento y generando un mayor gasto energético (Storr 1976). La escasa influencia del oleaje y la corriente en el interior de la ensenada de Playa Mero, generó un ambiente casi constante pero más desfavorable que el que caracterizó a Cayo Sombrero.

A su vez las condiciones ambientales en Punta Brava fueron catalogadas de "severas y casi constantes". Este sitio está sometido a la acción continua del oleaje, además presentó una elevada turbidez, influida probablemente por la cercanía del pueblo de Tucacas y las desembocaduras de los Ríos Aroa y Yaracuy. El valor de la corriente fue similar al registrado para Playa Mero, aunque este arrecife está expuesto, por lo que quizás la influencia resultó algo mayor. Estas condiciones generaron un ambiente más tenso que en las otras dos localidades, mucha acción mecánica por parte del oleaje, lo cual se reflejó en la dominancia exclusiva de la especie incrustante excavadora Cliona aprica sobre los esqueletos de Acropora palmata en las aguas someras, con una cobertura lineal mayor que la observada en Playa Mero. Estas condiciones se mantienen sin muchas variaciones en el tiempo, debido a que la costa venezolana no es frecuentada por huracanes, aunque pueden ocurrir tormentas ocasionales en determinadas épocas del año o lluvias abundantes.

El evento de mortalidad masiva ocurrido en Morrocoy en enero de 1996 pudo haber influido en la composición de las comunidades de esponjas estudiadas. El factor que provocó este evento actuó sobre las comunidades bentónicas durante un corto período de tiempo, lo que redujo casi a cero la cobertura coralina en la mayoría de los arrecifes, con excepción de Cayo Sombrero y Cayo Pescadores. Probablemente la fuerte corriente que predomina en estos arrecifes redujo el tiempo de estadía del elemento perturbador, minimizando su efecto (Laboy-Nieves et al. 2001).

Particularmente en Playa Mero se realizaron estudios antes y después de este evento, reportándose un porcentaje de cobertura coralina previo de $36.56 \%$ y porcentajes de cobertura de esponjas de $1.52 \%$. Después de la mortalidad la cobertura coralina viva se redujo a $4.84 \%$ y la de esponjas a $0.53 \%$ (Villamizar 2000).

Analizando esta situación es comprensible que Cayo Sombrero sea una comunidad más diversa y con un mayor número de especies. Para la fecha de realización de este estudio, es posible inferir una recuperación en las comunidades de poríferos en las otras dos localidades, fundamentalmente en Playa Mero, pues aún en 1997 los porcentajes de cobertura de poríferos continuaban siendo bajos. Es lamentable no contar con datos de la estructura comunitaria de poríferos previos a la mortalidad, sin embargo, es muy probable que ante el incremento en la sedimentación y la turbidez que genera la muerte de la mayoría de los corales, hayan colonizado el espacio vacío las especies de poríferos que más se adaptan a estas condiciones junto a los octocorales, ya que los corales escleractíneos requieren de condiciones más favorables y son de crecimiento más lento. Hubiese sido interesante conocer la estructura de la comunidad de poríferos en cada arrecife y su relación con las variables ambientales. Esto hubiese permitido discernir el peso del evento de mortalidad en las diferencias observadas.

Este estudio intentó explicar la estructura comunitaria de las esponjas en base a la 
variación espacial de las condiciones ambientales observadas en el área de estudio para el momento en que este se llevó a cabo. Sin embargo, el evento de mortalidad que ocurrió en 1996, muy probablemente contribuyó a las diferencias encontradas entre las comunidades estudiadas, posiblemente a consecuencia de un efecto diferencial en los tres sitios.

En razón de lo expuesto, frente a la amenaza de cambios climáticos y la creciente intervención humana, se hace imprescindible la implementación de este tipo de estudios en ecosistemas tan sensibles a las perturbaciones como los arrecifes coralinos; particularmente en los Parques Nacionales cercanos a asentamientos humanos e industrias y en otros espacios marino costeros tanto del país como de la región.

\section{AGRADECIMIENTOS}

Este trabajo fue realizado en el marco del macroproyecto Agenda Parque Nacional Morrocoy, subproyecto № 96001 754, financiado por FONACIT. Los autores agradecen a Juliana Fernández por la corrección final y a Dolores González Arnay del Centro de Estudios Integrales del Ambiente de la Universidad Central de Venezuela (CENAMB-UCV) por su valioso aporte en ideas y correcciones.

\section{RESUMEN}

El presente estudio pone en evidencia la variación en la estructura de la comunidad de esponjas en tres localidades del Parque Nacional Morrocoy (Cayo Sombrero, Playa Mero y Punta Brava) con distintas condiciones ambientales y afectadas diferencialmente por una mortalidad masiva ocurrida en 1996. En un total de 15 transectos de $10 \mathrm{~m}$ de largo y $1 \mathrm{~m}$ de ancho en cuatro estratos comprendidos entre 3 y $15 \mathrm{~m}$ de profundidad en cada localidad; se contabilizaron los individuos por especie para calcular la abundancia relativa, diversidad y equidad. Se analizaron las variables ambientales por localidad y se aprecian diferencias entre ellas con respecto a la corriente, turbidez, y exposición al oleaje. Fueron totalizadas 27 especies; Cayo Sombrero (23), Playa Mero (18) y Punta Brava (15), en la primera localidad domina: Agelas sceptrum, Amphimedon erina y Niphates erecta, en Playa Mero: Niphates erecta y Dysidea etherea y en Punta Brava: Dysidea etherea, Niphates erecta y Amphimedon erina. La composición de especies mostró diferencias estadísticamente significativas entre localidades. La mayor diversidad y equidad correspondió a Cayo Sombrero; donde las condiciones fueron más favorables para el crecimiento de estos organismos, seguido de Playa Mero y Punta Brava, guardando las dos primeras mayor similitud entre sí, según Índice de Sorensen.

Palabras clave: comunidad de esponjas, Parque Nacional Morrocoy, composición de especies, variables ambientales, evento de mortalidad masiva.

\section{REFERENCIAS}

Aerts, L.A.M. 1998. Sponge/Coral interactions in Caribbean reefs: analysis of overgrowth patterns in relation to species identity and cover. Mar. Ecol. Prog. Ser. 175: 241-249.

Alcolado, P.M. 1992. Sobre la interpretación del ambiente marino mediante el empleo de los índices de diversidad y equitatividad. Cien. Biol. 24: 124-127.

Alcolado, P.M. 1999. Comunidades de esponjas de los arrecifes del Archipiélago Sabana-Camagüey, Cuba. Bol. Invest. Mar. Cost. 28: 95-124.

Álvarez, B. \& M.C. Díaz. 1985. Las esponjas de un arrecife coralino en el Parque Nacional Archipiélago de Los Roques: Taxonomía y ecología. Tesis de grado, Universidad Central de Venezuela, Caracas, Venezuela.

Álvarez, B., M.C. Díaz \& R.A. Laughlin. 1990. The sponge fauna on a fringing coral reef in Venezuela I: Composition, distribution and abundance, p. 368-366. In K. Rützler (ed.). New perspectives in sponge biology. Smithsonian Institution, Washington D.C, EE.UU.

Alvizu, A. 2006. Estructura comunitaria de esponjas asociadas a dos parches coralinos en la Isla de Cubagua, Venezuela, durante el año 2005. Tesis de Grado, Universidad de Oriente, Boca del Río, Estado Nueva Esparta, Venezuela.

Amaro, M. \& I. Liñero. 2002. Demospongiae (Porifera) de Isla Larga, Bahía de Mochima, Venezuela. Bol. Inst. Oceanogr. Venezuela, Univ. Oriente 41: 45-53.

Amaro, M. \& I. Liñero. 2006. Esponjas más comunes en ambientes someros (Porifera: Demospongiae) de la Bahía de Mochima, Estado Sucre, Venezuela. Bol. Inst. Oceanogr. Venezuela, Univ. Oriente 45: 109-125.

Anderson, M.J. 2001. A new method for non-parametric multivariate analysis of variance. Aust. Ecol. 26: 32-46.

Bell, J.J. \& D.K.A. Barnes. 2000. A sponge diversity centre within a marine 'island'. Hydrobiologia 440: 55-64.

Butler IV, M.J., J.H. Hunt, W.F. Herrnkind, M.J. Childress, R. Bertelsen, W. Sharp, T. Matthews, J.M. Field \& H.G. Marshall. 1995. Cascading disturbances in 
Florida Bay, USA: cyanobacteria blooms, sponge mortality, and implications for juvenile spiny lobsters Panulirus argus. Mar. Ecol. Prog. Ser. 129: 119-125.

Chaves-Fonnegra, A., V. Mateo-López \& S. Zea. 2005. Ecología química de las esponjas excavadoras Cliona aprica, C. caribbaea, C. delitrix y C. tenuis. Bol. Invest. Mar. Cost. 34: 43-67.

Clarke, K.R. \& R.M. Warwick. 2001. Change in marine communities: an approach to statistical analysis and interpretation. Plymouth Marine Laboratory, Plymouth, Reino Unido.

Díaz, H., M. Bevilacqua \& D. Bone. 1985. Esponjas en manglares del Parque Nacional Morrocoy. Fondo Editorial Acta Científica Venezolana, Caracas, Venezuela.

Díaz, M.C., B. Álvarez \& R.W. Van Soest. 1987. New species of Demospongiae (Porifera) from the National Park "Archipiélago de los Roques", Venezuela. Bijdragen tot de Dierkunde 57: 31-41.

Díaz, M.C. \& K. Rützler. 2001. Sponges: An essential component of Caribbean coral reef. Bull. Mar. Sci. 69: 535-546.

Erwin, M.P. \& R.W. Thacker. 2007. Incidence and identity of photosynthetic symbionts in Caribbean coral reefs sponges assemblages. J. Mar.Biol. Ass. U.K. 87: 1683-1692.

Hooper, J.N.A. \& van Soest, R.W.M. 2002. Systema Porifera: A guide to the classification of sponges. Kluwer Academia, Plenum Publishers, Nueva York, EE.UU.

Hubbard, R. 1990. A sessile shallow-water community dominated by sponges and algae at Nelson Island, Trinidad and Tobago. Carib. Mar. Stud. 1: 152-158.

Laboy-Nieves, E.N., E. Klein, J.E. Conde, F. Losada, J.J. Cruz \& D. Bone 2001. Mass mortality of tropical marine communities in Morrocoy, Venezuela. Bull. Mar. Sci. 68: 163-179.

Losada, F. \& J.A. La Schiazza. 1989. Caracterización biológica de algunas comunidades marinas en el Parque Nacional Morrocoy, p. 305-344. In D. Pérez de Acosta (ed.). Línea Base de Referencia Biológica en el Ambiente Marino Costero del Área de Golfo Triste. Informe Final de Proyecto por Contrato PEQUIVEN - USB, Caracas, Venezuela.

Magurran, A.E. 1988. Ecological Diversity and Its Measurement. Princeton University, Princeton, Nueva Jersey, EE.UU.

Martín, A. 2005. Evaluación de los parámetros físicoquímicos en el Parque Nacional Morrocoy y zonas aledañas, Venezuela, p. 1-56. In D. Bone (ed.). Estudio integral del sistema Parque Nacional Morrocoy con vías al desarrollo de planes de uso y gestión para su conservación. Informe Final, USB-UCV-UNEFMINIA-FONACIT, Caracas, Venezuela.

McArdle, B.H. \& M.J. Anderson. 2001. Fitting multivariate models to community data: a comment on distance based redundancy analysis. Ecology 82: 290-297.
Mckenna, S.A. 1997. Interactions between the boring sponge Cliona lampa and two hermatipic corals from Bermuda. Proc. $8^{\text {th }}$ Int. Coral. Reef. Sym. 2: 1369-1374.

Nuñez, M., J.G. Rodriguez \& M.C. Díaz. 2010. Distribución de esponjas (Porífera) a lo largo de un gradiente de profundidad en un arrecife coralino, Parque Nacional San Esteban, Carabobo, Venezuela. Rev. Biol. Trop. 58: 175-187.

Olivares, M.A. 1976. Estudio taxonómico de algunas Demospongiae (Porífera) de la Bahía de Mochima, Edo. Sucre, Venezuela. Trabajo de Ascenso, Universidad de Oriente, Cumaná, Venezuela.

Pauls, S.M. 1998. Estudio sistemático y biodiversidad de Porífera y Cnidaria en la Bahía Ciénaga de Ocumare de la Costa. Trabajo de Ascenso, Universidad Central de Venezuela, Caracas, Venezuela.

Pielou, E.C. 1966. The measurement of diversity in Different Types of Biological Collections. J. Theor. Biol. 13: 131-144.

Rützler, K. 1978. Sponges in Coral Reef. Coral reefs: Research Methods, p. 299-313. In D.R. Stoddart \& R.E. Johannes (eds.). Monographs on Oceanographic Methodology, UNESCO, París, France.

Sará, M. 1970. Competition and Cooperation in Sponge Populations, p. 273-284. In W.G. Fry (ed.). The biology of the Porifera, Symp. Zool. Soc. London. Academic, Londres y Nueva York, EE.UU.

Solana, P., B. Castellanos \& M. Nalesso. 2004. Medición de variables hidrodinámicas y ambientales en Morrocoy, Venezuela. Rev. Téc. Ing. Univ. Zulia 27: 100-113.

Sorensen, T. 1948. A method of stabilizing group of equivalent amplitude in plant sociology based on the similarity of species contents and its aplications to analysis of the vegetation of Danish Commons. K. Danska Vid. Selsk. Bot. Srk. 5: 1-34.

Storr, J.F. 1976. Ecological factors contrilling sponge distribution in the Gulf of Mexico and the resulting zonation, p. 261-276. In F.W. Harrison \& R.R Cowden (eds.). Aspects of Sponge Biology. Academic, Londres y Nueva York, EE.UU.

Valderrama, D. \& S. Zea. 2003. Esquema de distribución de esponjas arrecifales (Porifera) del noroccidente del Golfo de Urabá, Caribe Sur, Colombia. Bol. Invest. Mar. Cost. 32: 37-56.

Villamizar, E. 1985. Fauna asociada a las esponjas Aplysina archeri y Aplysina lacunosa a lo largo de un gradiente de profundidad en el Parque Nacional Archipiélago de Los Roques. Tesis de grado, Universidad Central de Venezuela, Caracas, Venezuela.

Villamizar, E. 2000. Estructura de una comunidad arrecifal en Falcón, Venezuela, antes y después de una mortalidad masiva. Rev. Biol. Trop. 47: 19-30.

Zea, S. 1987. Esponjas del Caribe Colombiano. Catálogo Científico, Bogotá, Colombia. 
